\title{
Design and Application of Teaching about Network Course Education based on Cloud Computing
}

\author{
Duanli Yang,Tao Guo, Liguo Zhang \\ College of Information Science and Technology, \\ Agricultural University of Hebei, Baoding 071001,China \\ 2093363847@qq.com
}

\begin{abstract}
Network cloud computing has become a convenient tool for people to continuous learning, it provides conditions to engage in scientific research for our new generation of sports workers future employment and development. In this paper, a cloud computing model was established, and research and development of network course education based on cloud computing is a kind of practice and the accumulation of the technology, which can develop a perfect function, with good interactive performance, can solve various problems of physical training. And the effect of prediction model time is more close to actual time, can efficiently limit the possibility of falling into local convergence, the optimal solution's time of objective function value is shorten which meet the student's needs.
\end{abstract}

Keywords: Network cloud computing; network course education; Interactive performance

\section{Introduction}

Cloud computing is a now wildly used architecture hot, it's product of the development of grid computing, distributed computing, network storage and parallel processing [1]. It shows that the student's applications can operate without personal computer but the server cluster in the Internet. There are three basic forms of cloud computing services including: Infrastructure as a Service (IAAS), Platform as a Service (PAAS) and Software as a Service (SAAS) [2]. In cloud computing, the allocation of resources is a very important issue, the unsatisfactory allocation of resources can easily led the cloud's servers crashed and other servers in idle. So in cloud environment, the problem mostly need to solve is the ways to control any server's resources allocation and use condition by the information communication of local and in the Internet to make better use of the resources. We can share the network information resources, rapid evolution and the development of the Internet technology and the popularity of various regions in the world scope continents, to learn to use the network will become an important way in the future education and learning areas, Internet applied to teaching and learning will become our future study and the inevitable trend of the development of our life learning.

\section{The Definition of Cloud Computing and Cloud Education}

Cloud computing is a new calculation mode making virtual technology as its kernel. It inherits and develops distributed processing, parallel processing and grid computing and delivers infrastructure, development platform and software as a kind of services to users in need. As shown in Figure 1. 


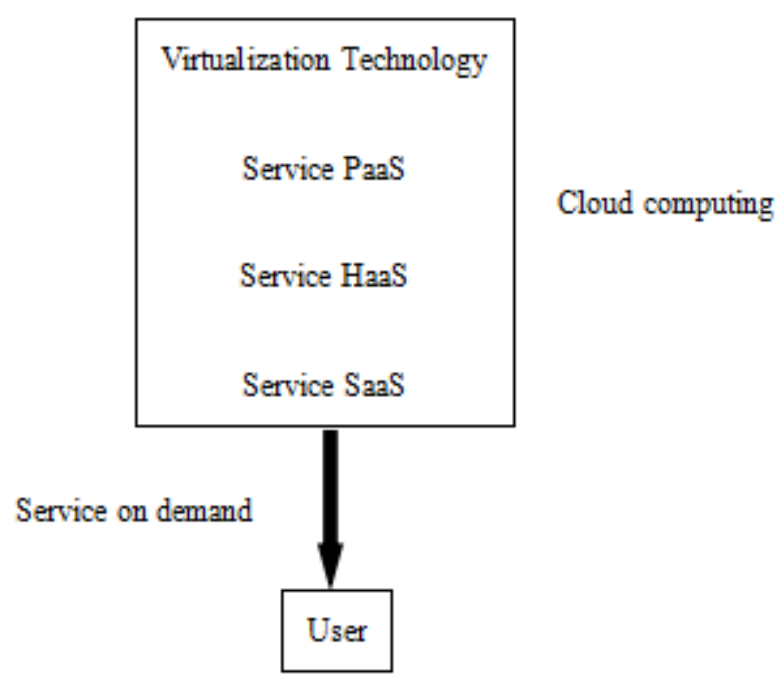

Figure 1. Diagram of Cloud Computing

\section{The Definition of Cloud Education}

Cloud education is to realize the distribution management and sharing application of teaching resources, satisfy teachers with teaching model innovation and meet the need of individualized learning by using the idea and technical pattern of cloud computing[3-5]. Users can interactively study and communicate on the internet in real time only with a browser. Teachers and students can log in the platform of cloud education to obtain resources with a account number and a browser. Cloud education can efficiently resolve the problem of low sharing of educational information resources. Tao Xiping, a committee member of National Education Counseling Agency, says that cloud education is to link all educational resources together and formulate a sharing of education resource library by virtue of the core technology of cloud computing. Shown in Figure 2.

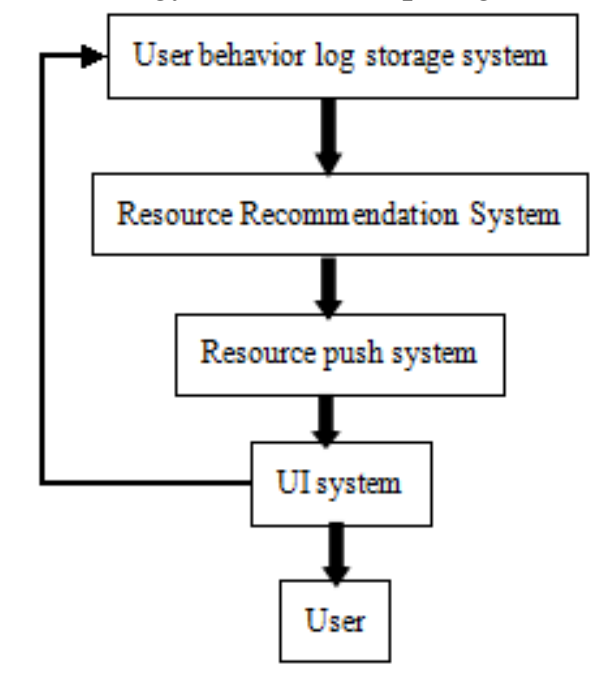

Figure 2. Diagram of Cloud Education

Learning that is based on cloud environment (such as micro-class, cloud computing terminals, video communication and other social learning communication software), is the existence of the common problems during the process of teachers' teaching and students' learning, which can take a variety of forms of organization to have interaction, discussion and experience sharing, so as to achieve solving the network practical problems. The 
specific learning mode can be shown in Figure 3.

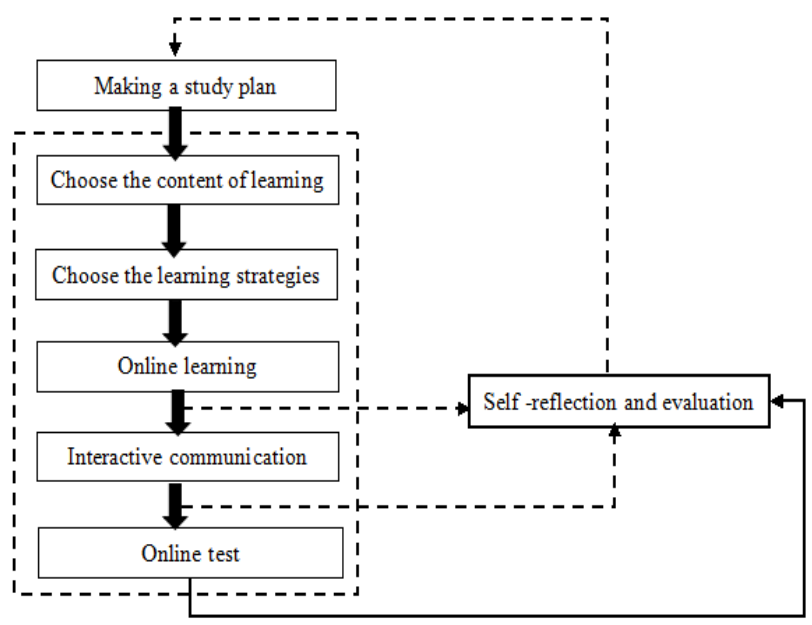

Figure 3. Learner's Autonomous Learning Model under Cloud Computing Cloud Environment

\section{The Basic Connotation of Cloud Computing Learning}

C-learning is a way of e-learning in a cloud computing learning situation. It sends learning resources to cloud computing terminal equipments such as PDA, 3G phones, Ipad and so on by relying on cloud computing communication network and wireless network in order to guarantee learners to study at any time and any place. In other words, C-learning brings learning environment to people in any space and time instead of attracting them into a fixed learning environment.

\section{The Essence of Cloud Computing Learning}

C-learning has its mobility and situationality. It possesses four features: first, it needs the support of wire less cloud computing network; second, learners are out of the control of space and time and they are in a cloud computing situation; third, , it needs the technical support of cloud computing interconnection technique; fourth, teachers and students can make both-way and free interaction. To sum up, seen in Figure 4. The author believe that learning style conforming to cloud computing learning must have three features: first one is digitized teaching content; second one is cloud computing media as learning tools; third is that teachers and students must be in separated time and space, which are the criteria of cloud computing learning.

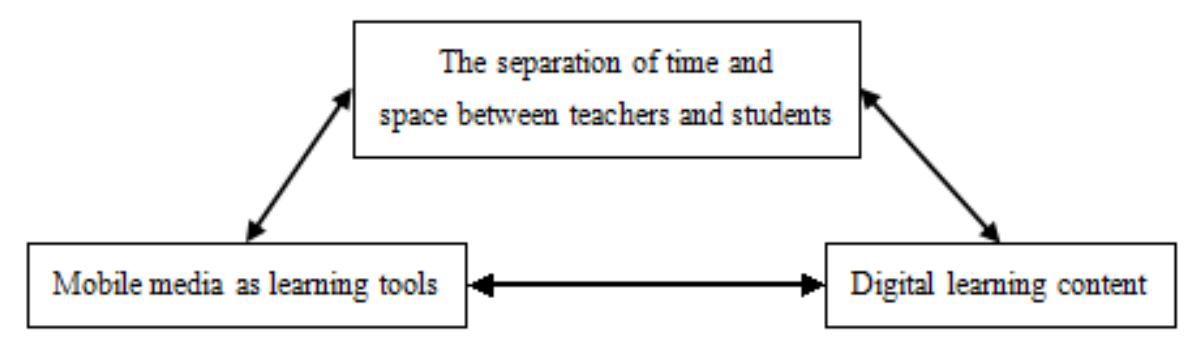

Figure 4. Three Necessary Criteria of Cloud Computing Learning 


\section{Basic Knowledge of Cloud Model}

The cloud model is a transformation model uses linguistic values to express the uncertainty between a certain conception and its quantification expression, it fully combines fuzziness and randomness and forms the mapping between qualitative and quantification, shows in Figure 5.

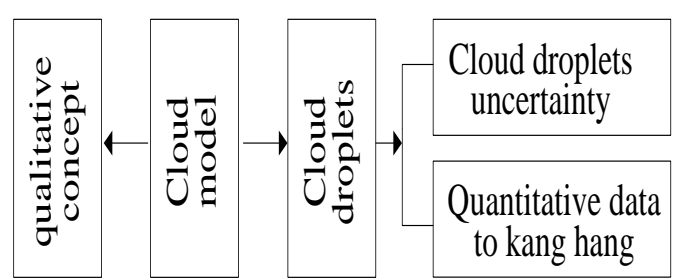

Figure 5. Transformation Schematic of Cloud Mode's Qualitative Concept and Quantitative Data

Sets $\mathrm{U}$ is discourse domain expressed by accurate numerical value, $\mathrm{A}$ is corresponding qualitative concept in U. If quantitative value $x \in u$ and $\mathrm{x}$ is a random implementation with likely normal distribution of qualitative concept $\mathrm{A}$ in discourse domain $\mathrm{U}$, the certainty degree $A(x) \in[0,1]$ of $\mathrm{x}$ to $\mathrm{A}$ is also a random number with likely normal distribution, then data array $\left(x, A\left(x_{i}\right)\right)$ is called as cloud drop, the whole element $x_{i}(i=1,2 \ldots n)$ in discourse domain $\mathrm{U}$ and its certainty degree $A\left(x_{i}\right)$ for $\mathrm{A}, i, e, \mathrm{n}$ data array $\left(x, A\left(x_{i}\right)\right)$, forms the cloud model with $\mathrm{n}$ cloud drop, calls $\mathrm{x}$ distribution in discourse domain $U$ as cloud distribution. The number characteristics of cloud model are expressed as expectation (Ex), entropy (En) and excess entropy (He). Among them, expectation (Ex) refers to the central value of discourse domain $U$, is the center of qualitative concept, reflects the cloud focus of the whole cloud drop swarm; entropy (En) refers to the range which can be received by fuzzy concept, $E n \succ 0$; excess entropy (He) is a uncertain measurement of entropy, i,e, the excess entropy is the entropy's entropy, ${ }_{\mathrm{H}}>0$. The excess entropy reflects the degree of reach an agreement of cloud drop of representation qualitative concept or the concentration degree of cloud drop's representation qualitative concept; the bigger excess entropy is, the qualitative concept has worse common sense or the qualitative concept is worse decentralization.

\section{Design Principles of Network Course of Cloud Computing}

The basic knowledge of volleyball, technology, skills and tactics are used in competition and rules of network course match and the cloud teaching theory.

the network principle, it is not restricted by time and space, sharing and integration with various resources, network cloud computing must be able to through this network platform to transfer a large amount of learning information modernization, but also through the computer terminal to the use of and access to cloud computing content [6]. Must have its own position with space and realm name, the only way to ensure the function of cloud computing, network teaching for a greater degree to replace the existing ordinary teaching will be the future, meanwhile, accelerated the information to further develop the technical level and technical innovation, further improve the overall quality of teachers, teaching the concept of further update and so on. Prospects for the development of network cloud computing is broad, driven by the software on the network, to achieve a more extensive development of nationwide fitness campaign, the network is essential.

The principle of autonomy. People there are individual differences in terms of cognition, unique methods and habits have different cognitive learning styles on the outside of the information. The independent performance of cloud computing should 
allow the student according to the ir own learning goals, learning to arrange the learning progress and learning time and can learn the main content specific to each training process, and the student can control the information features video, at any time to play music, fully reflects the students' learning autonomy, and has the advantage of flexibility cloud computing, changed the traditional teacher teaching and classroom centered teaching mode in the past to a great extent, thus forming a student-centered teaching mode, such teaching mode is more advantageous to teach students in accordance of their aptitude and the implement individualized teaching goal.

In cloud computing environment, the mostly used model is Map/Reduce, this model operates well in large-scale parallel task. Especially in cloud computing environment, it needs to processes each cloud student's resource number, time, network channel fee, etc. in time. The currently related task scheduling algorithm focuses on the needs of overall task, considers less about the cloud student's complementing time, which led to unreasonable in time and resources distribution for the students when multiple tasks operates. Supposes cloud client's tasks of cloud computing as Table 1:

a) Divides large-scaled task into relatively small tasks, divides in average, the sub-tasks' operating time are similar.

b) The number of resource distribution offers enough for sub-tasks.

c) Reasonable defines sub-task occupies resources time.

Table 1. Sub-tasks and Resources Table

\begin{tabular}{l|l|l|l|l}
\hline $\begin{array}{l}\text { The } \\
\text { subta } \\
\text { sks }\end{array}$ & $\begin{array}{l}\text { resou } \\
\text { rces }\end{array}$ & $\begin{array}{l}\text { Running } \\
\text { time }\end{array}$ & Running costs & $\begin{array}{l}\text { Total } \\
\text { resources }\end{array}$ \\
\hline$n_{1}$ & $m_{1}$ & $t\left(n_{1}, m_{1}\right)$ & $\cos t\left(n_{1}, m_{1}\right)$ & \\
\hline$n_{2}$ & $m_{2}$ & $t\left(n_{2}, m_{2}\right)$ & $\cos t\left(n_{2}, m_{2}\right)$ & \multirow{2}{*}{$S m=\sum_{i=1} m_{i}$} \\
\hline$\ldots \ldots$ & $\ldots \ldots$ & $\ldots \ldots \ldots \ldots$ & $\ldots \ldots \ldots$ & \\
\hline$\ldots$ & $\ldots \ldots$ & $\ldots \ldots \ldots$ & $\cos t\left(n_{i}, m_{j}\right)$ & \\
\hline$n_{n}$ & $m_{n}$ & $t\left(n_{i}, m_{j}\right)$ & & \\
\hline
\end{tabular}

$N_{i}$ refers to the number of sub-tasks, ${ }^{m_{i}}$ refers to the number of resources, $t\left(n_{i}, m_{i}\right)$ refers to the time in task ${ }^{i}$, resources ${ }^{j},{ }^{\cos \left(n_{i}, m_{j}\right)}$ refers to the costs in task ${ }^{i}$, resources ${ }^{j}$. In these above models, supposes the resources in cloud computing reasonable can be distributes into the computing resources of sub-tasks and ensures the shortest time and the lower costs for complementing the sub-tasks.

The overall diversity and difference principle of a high quality cloud computing should be a holistic and contains a variety of cyber source information, the newer and better information has been added to the making of information, but also contains a large number of unique properties different from other information, always can let students like and love.

Interactive principle of interaction between information content and student's most important link, is the important difference between a network cloud computing with traditional teaching means, the network cloud computing developed in full consideration of who is the main object, fully in each plate block design as far as possible, adding more evaluation and interaction. 


\section{Mathematical Model}

The resources distribution under cloud computing related some specification for cloud students, they have this restrictions as following in general:

a) The required order is given for each cloud student.

b) Can only receive one requirement of one student in one period of time, each student can only be occupied by one server in cloud server, one started it cannot be disrupted.

c) Each task can only operate once in one cloud server in the whole machining process.

d) Without considering the superiority of each task.

The mathematic model of this problem can be expressed as following:

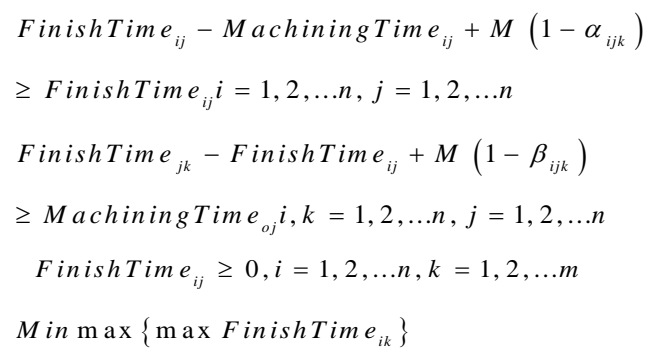

Formula (1) refers to the operating order of each sub-task determined by each task. Formula (2) refers to the order of each sub-task, formula (3) refers to each sub-task's time variable restriction. Formula (4) refers to objective function. FinishTime $e_{i j}$ refers to the complementing time of task $i$ in server $\mathrm{k}$, MachiningTime $_{o j}$ refers to the processing time of task $i$ in server $\mathrm{k}, \mathrm{M}$ is a coefficient defined values, $\alpha_{i j k}$ and $\beta_{i j k}$ are expressed as following: $\alpha_{i j k}$ is 1 refers to server $\mathrm{j}$ operating task $i$ in server $\mathrm{k}, \alpha_{i j k}$ is 0 refers to other conditions. $\beta_{i j k}$ is 1 refers to task $i$ processing task $i$ in server $\mathrm{k}, \beta_{i j k}$ is 0 refers to other conditions.

To define the Multi-modal network model by the undirected graph $\mathrm{G}=(\mathrm{V}, \mathrm{E}, \mathrm{W})$, in which $\mathrm{V}$ can be presented as a set of vertices, E can be presented as the edge of graph, W can be presented as the weighting set, namely the network distance that related to the edge. For any $\mathrm{v} \in \mathrm{V}, \mathrm{v}$ refers to the road-intersection in the network or the boundary point of network graph. The edge $\left(v, v^{\prime}\right) \in E$ refers to the Multi-modal in the network, and the weight $w \in W$ refers to the length of a road, namely $\left|v, v^{\prime}\right|$, due to the undirected graph, the distance of the Multi-modal is a two-way, that is to say $\left|v, v^{\prime}\right|=\left|v^{\prime}, v\right|$.

\section{Resource Object Descriptive Modeling}

When define the space object set $\mathrm{P}$, it is always on the edge $\mathrm{E}$ of the Multi-modal graph $\mathrm{G}$ for any $\mathrm{p} \in \mathrm{P}$. P was defined as a 3-tuple ( $\mathrm{p} .1$, p.d, p.a), respectively indicating the location of resources, description text and capability information. The size of the space object set was represented with ${ }^{|P|}$. For the distance from object $\mathrm{p}$ to the two end points, it can be represented with ${ }^{\mid p, v} \mid$ and $\left|p, v^{\prime}\right|$ respectively, where $\mathrm{v}$ and $\mathrm{v}^{\prime}$ is the two end points on the edge of $\left(\mathrm{v}, \mathrm{v}^{\prime}\right)$ where object $\mathrm{P}$ are located . In the Multi-modal network graph G, the distance between object $\mathrm{p}$ and $\mathrm{p}^{\prime}$ was represented with $\left\|p, p^{\prime}\right\|$. Meanwhile, in the set of edges $\mathrm{E},\left(\mathrm{v}, \mathrm{v}^{\prime}\right)$ and $\left(\mathrm{v}^{\prime}, \mathrm{v}\right)$ is the same, and the distance of $\left.\right|^{|p, v|}$ equals $\left|v, v^{\prime}\right|-\left|p, v^{\prime}\right|$. 
Therefore, the distance from $\mathrm{P}$ to another endpoint can be achieved once you know the distance from $P$ to the endpoint.

\section{Measurement about Cloud Model}

The cloud model's spray characteristic refers to the character of cloud drop distributes around cloud expectation curve's discrete degree[7]. Professor Liuyu, etc. made researches on excess entropy measures cloud drop's discrete degree with fixed entropy. But these works did not show the essence factors of determine cloud model's spray characteristic, $i, e$, the standard deviation Y's distribution of cloud drop quantitative data $\mathrm{X}$ determines the cloud model's spray characteristic. The same as the cloud distribution probability density of cloud model algorithm identified is the theoretical basis of uncertainty reverse cloud model algorithm, this chapter revised the cloud distribution probability density and gave a strict proof according to spray characteristic $\mathrm{Y}>0$.

The positive direction cloud model algorithm steps in one-dimension theory's domain are as following:

Step 1: Generates normal random number $y_{i}$ whose expectation is $E_{n}$, standard deviation is $\mathrm{He}$;

Step 2: Generates normal random number $x_{i}$ whose expectation is Ex, standard deviation is $y_{i}, x_{i}$ is a concrete and quantitative realize of qualitative concept $\mathrm{A}$ operates in its corresponding quantitative theory of the domain U, called cloud drop qualitative data;

Step 3: Calculates $r_{i}=\exp \left(-\frac{\left(x_{i}-E_{x}\right)^{2}}{2 y_{i}^{2}}\right), r_{i}$ is the certainty degree or subjection degree of $x_{i}$ belongs to qualitative concept A;

Step 4: Repeats step one to three until generates n cloud.

Prove: because $y-r\left(E_{n}, H_{e}{ }^{2}\right)$, En refers to the discourse domain must be greater than zero, as $x \square N\left(E_{x}, y^{2}\right), y$, as the standard deviation of $x$, must be greater than zero, so according to normal distribution random variable meets $3^{\sigma}$ rule, gets $E_{n} / H_{e} \geq 3$. Besides, the probability density of $\mathrm{Y}$ is

$x_{i}(y)=\frac{1}{\sqrt{2 \pi H_{e}}} \exp \left\lfloor-\frac{\left(t-E_{n}\right)}{2 H e^{2}}\right\rfloor$

When $x_{i}=y$, the conditional probability density is

$x_{i, j}(x \mid y)=\frac{1}{\sqrt{2 \pi y}} \exp \left[-\frac{\left(x-E_{x}\right)^{2}}{2 y^{2}}\right]$

Gets joint probability density through conditional probability density formula:

$x(i, j)=\frac{1}{2 \pi H_{e} j}-\exp \left[-\frac{\left(j-E_{n}\right)^{2}}{2 H e^{2}}-\frac{(i-E i)^{2}}{2 j^{2}}\right]$

Gets probability density which marginal probability density is cloud distribution through joint probability density formula:

$x_{i}(x)=\int_{x}^{x} \frac{1}{2 \pi H e y} \exp \left[-\frac{(y-E n)}{2 H e}-\frac{\left(x-E_{x}\right)}{2 y^{2}}\right]$

This formula has no analytic form Quod $x_{i}$ demonstrandum.

From step 2, 3, $y$ is the standard deviation of cloud drop qualitative data $X$, its distribution character directly determines the cloud drop's distribution character, the bigger distribution scale of Y, the more cloud drop distributes discrete. Because 
$Y \sim N\left(E n, H e^{2}\right)$

This text takes $a=E n / H e$ as the measurement of cloud drop's discrete degree, called spray factor, because qualitative data's standard deviation Y, En and $\mathrm{He}$ must be greater than zero at the same time so $a \geq 3$. Spray factor $a$ integrative considers the nature that standard deviation $\mathrm{Y}$ of cloud drop's qualitative data $\mathrm{X}$ must be greater than zero, the distribution of $\mathrm{Y}$ directly affects cloud drop discrete degree and a determines the distribution character of $\mathrm{Y}$, so 0.0 can be the significant digital characteristic of cloud model to presents the discrete condition of cloud drop's distribution. The spray characteristic of cloud model has the following characters:

Character 1: The distribution characteristics of cloud drop's qualitative data standard deviation determines the cloud drop's distribution characteristics, a refers to the cloud drop's discrete degree and $a \geq 3$. The smaller $a$ be, the bigger discrete degree of cloud drop's distribution; when $\alpha=3$, the discrete degree of cloud drop's distribution reaches the biggest; the bigger $\alpha$ is, the smaller discrete degree of cloud drop's distribution, finally tends to normal distribution. Now the cloud drop all approximate distributes on cloud expectation curve.

Character 2: cloud distribution's corresponding range of spray factor: $3 \leq a \leq 18$.

The spray factor determines the distribution characters of cloud drop qualitative data, and the kurtos is describe the figure of data distribution at the same time, the kurtosis of normal distribution is 3 , if the kurtosis of cloud distribution values around 3, the cloud distribution turns to normal distribution.

\section{Conclusion}

Cloud computing is a new-style kind of teaching model, innovation and development is also the modern teaching mode, has powerful function to promote the development of Internet, make the power of the cloud computing is more and more big, a cloud computing requires producers not only has the course of professional know ledge, but also must have the comprehensive use of computer software, and education science, psychology, aesthetics and other subject knowledge to do support, information R \& D needs to use engineering, science, literature and complement each other's advantages of multi subjects. Development and use of the cloud computing, can add features to high education, so that the student can play the main role, using the network function complement each other, improve learning efficiency; influence to the field of physical education in a positive, it will also become one of the most powerful tools of adolescent physical training and learning in the new century.

\section{References}

[1] Q. Zhang, B. Li, "Discriminative K-SVD for dictionary learning in face recognition”, Proceeding(s) of Conference on Computer Vision and Pattern Recognition (CVPR), pp. 2691-2698, (2010).

[2] X Mao. Nguyen, Q M. Le, V Pham, T Tran, B H. Le, "Multi-scale Sparse Representation for Robust Face Recognition", Proceeding(s) of Conference on Knowledge and Systems Engineering (KSE), pp. 195-199, (2011).

[3] Y. Zhai, J. Gan, J. Li, "Study of occluded robust face recognition approach based on homotopy algorithm and color information fusion", Signal Processing, vol. 21, no. 11, pp.1762-1768, (2011).

[4] S. Li, Y. Geng, J. He, K. Pahlavan, "Analy sis of Three-dimensional Maximum Likelihood Algorithm for Capsule Endoscopy Localization”, 2012 5th International Conference on Biomedical Engineering and Informatics (BMEI), Chongqing, China Oct. (2012), pp 721-725.

[5] Y. Geng, J. He, H. Deng and K. Pahlavan, "Modeling the Effect of Human Body on TOA Ranging for Indoor Human Tracking with Wrist Mounted Sensor", 16th International Symposium on Wireless Personal Network Communications (WPMC), Atlantic City, NJ, Jun. (2013).

[6] Y. Geng, J. He, K. Pahlavan, Modeling the Effect of Human Body on TOA Based Indoor Human Tracking[J], International Journal of Wireless Information Networks, vol. 20, no. 4, pp. 306-317

[7] L. Cordero-Grande, G. Vegas-Sánchez-Ferrero, P. Casaseca-de-la-Higuera, J.A. San-Román-Calvar, A. Revilla-Orodea, M. Martín-Fernández, C. Alberola-López, "Unsupervised 4D myocardium 
segmentation with a Markov Random Field based deformable model", Medical Image Analysis, vol.15, pp. 283-301, June 2011. http://dx.doi.org/10.1016/j.media. (2011).01.002

\section{Authors}

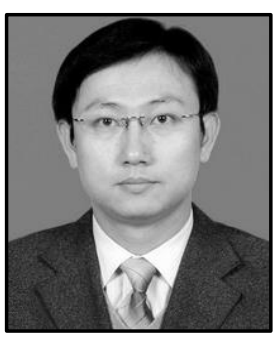

Duanli Yang, Current position, grades: Associate Professor, College of Information Science and Technology (China) Scientific interest: intelligent information processing, network course education. 
International Journal of Grid and Distributed Computing

V ol. 9, No. 3 (2016) 